\title{
Direct Contact between Human Peripheral Blood Mononuclear Cells and Renal Fibroblasts Facilitates the Expression of Monocyte Chemoattractant Protein-1
}

\author{
Lirong Hao $^{a}$ Hirokazu Okada $^{a}$ Yoshihiko Kanno $^{a}$ Tsutomu Inoue ${ }^{a}$ \\ Tatsuya Kobayashi ${ }^{a}$ Yusuke Watanabe ${ }^{a}$ Frank Strutz $^{b}$ Gerhard A. Müller ${ }^{b}$ \\ Hiromichi Suzuki a \\ aDepartment of Nephrology, Saitama Medical School, Saitama, Japan; 'bepartment of Nephrology and \\ Rheumatology, Georg August University, Göttingen, Germany
}

\section{Key Words \\ Inflammation · Fibroblasts · Peripheral blood mononuclear cells $\cdot$ Monocyte chemoattractant protein-1 - Intercellular adhesion molecule-1}

\begin{abstract}
Background: Cell-to-cell interaction is thought to be an important feature of a variety of biological processes. As far as the proinflammatory process is concerned, the interaction between mesangial cells and monocytes/ macrophages induces the expression of monocyte chemoattractant protein-1 (MCP-1), and this may play a role in glomerulonephritis. In this study, we investigated whether the cell-to-cell interaction between immune cells and renal fibroblasts induces MCP-1 gene expression, which may be involved in interstitial inflammation in the kidney. Methods: Human renal fibroblast cell lines, tNKF (from a normal kidney) and tFKIF (from a kidney with fibrosis), and peripheral blood mononuclear cells (PBMC) were used to assess the effect of cell-to-cell contact on the expression of MCP-1 mRNA in the fibroblasts. The expression of the MCP-1 gene in the fibroblasts was also examined after stimulation with tumor necrosis fac-
\end{abstract}

tor- $\alpha$ (TNF- $\alpha$ ) and the culture supernatant from PBMC. RT-PCR was used to detect MCP-1 mRNA expression. Neutralizing antibodies to intercellular adhesion molecule-1 (ICAM-1) and vascular endothelial adhesion molecule-1 (VCAM-1) were used to block the cell-to-cell contact between the fibroblasts and PBMC. Results: TNF- $\alpha$ and the culture supernatant from PBMC increased MCP-1 gene expression in tNKF cells. Contact culture with PBMC also significantly increased MCP-1 gene expression in tNKF cells. Although the basal level of MCP-1 mRNA was higher in tFKIF than tNKF cells, tFKIF cells did not respond significantly to any stimulation in this study. Following pretreatment with anti-ICAM-1 antibody, MCP-1 gene expression in tNKF cells was significantly suppressed in contact culture with PBMC. Anti-VCAM-1 antibody treatment had no effects. Conclusion: It is suggested that the interaction between renal fibroblasts and PBMC was mediated through direct contact and by secreted humoral factors. ICAM-1 on renal fibroblasts may be involved in the direct cell-to-cell interaction inducing MCP-1 gene expression, which seems to be involved in renal interstitial inflammation.

Copyright (C) 2003 S. Karger AG, Basel

Hiromichi Suzuki, MD, PhD

Department of Nephrology, Saitama Medical School

38 Morohongo, Moroyama-cho, Irumagun

Saitama 350-0495 (Japan)

Tel. +81 49276 1611, Fax +81 49295 7338, E-Mail iromichi@saitama-med.ac.jp
Fax + 41613061234

E-Mail karger@karger.ch

www.karger.com

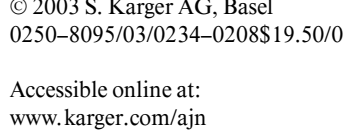




\section{Introduction}

Most forms of kidney disease lead to renal interstitial alterations consisting of immune cell infiltration, tubular atrophy, and fibrosis [1,2]. The degree of renal interstitial alterations is significantly correlated with functional outcome, regardless of the primary underlying disease $[1,3]$. The earliest feature of interstitial alterations is immune cell infiltration $[1,2]$. After attachment to and migration through the endothelium, following a chemokine gradient, recruited immune cells are supposed to interact with interstitial cells such as fibroblasts $[1,2,4,5]$. This interaction is mediated through a variety of adhesion molecules and their ligands [4-6], and likely starts proinflammatory cascades to facilitate further immune cell infiltration. Blaber et al. [5] and Clayton et al. [6] reported that leukocyte adherence to renal fibroblasts stimulated intercellular adhesion molecule-1 (ICAM-1) expression by fibroblasts, and those leukocytes also could trigger the synthesis of the chemokine RANTES by fibroblasts via ICAM- $1 / \beta_{2}$-integrin interaction. In addition to RANTES, monocyte chemoattractant protein-1 (MCP-1) has also been shown to play a pivotal role in the pathogenesis of glomerular and interstitial inflammation/fibrosis in the kidney [7-10]. Hisada et al. [11] reported that cell-to-cell interaction between mesangial cells and macrophages induced the expression of MCP-1 by mesangial cells. Therefore, in this study we investigated whether human peripheral blood mononuclear cells (PBMC) could induce MCP-1 gene expression in renal fibroblasts indirectly via humoral factors and directly via cell-to-cell contact.

\section{Material and Methods}

\section{Renal Fibroblast Culture}

Two renal fibroblast cell lines, tNKF and tFKIF, were established from a normal kidney and from a human kidney with fibrosis, respectively [12]. Both were maintained in DMEM (Gibco BRL, Grand Island, N.Y., USA) supplemented with 10\% FBS (Hyclone, Logan, Utah, USA), $2 \mathrm{~m} M L$-glutamine, $100 \mathrm{IU} / \mathrm{ml}$ penicillin, and $100 \mu \mathrm{g} / \mathrm{ml}$ streptomycin (Gibco BRL).

\section{Isolation of PBMC}

Peripheral blood samples were taken from Y.K., T.I., T.K. and Y.W. PBMC were isolated by centrifugation through Ficoll-Paque (Amersham Pharmacia Biotech, Uppsala, Sweden) density gradients according to the manufacturer's instructions, and suspended in RPMI 1640 medium (Gibco BRL) supplemented with 10\% FBS, $2 \mathrm{~m} M L$-glutamine, $100 \mathrm{IU} / \mathrm{ml}$ penicillin, and $100 \mu \mathrm{g} / \mathrm{ml}$ streptomycin. A small sample of isolated PBMC was stained with $0.04 \%$ trypan blue for $5 \mathrm{~min}$, and the cells excluding trypan blue were counted in a hemocytometer to evaluate viability.

Cell-to-Cell Interaction in Fibrogenesis
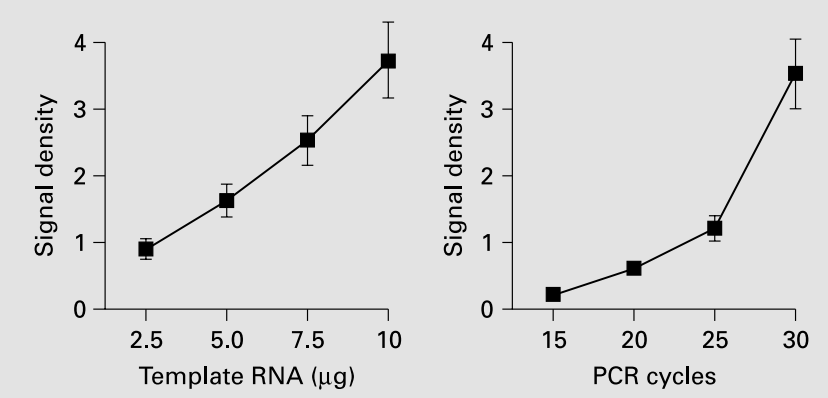

Fig. 1. Preliminary results of RT-PCR of MCP-1 gene expression with varying amounts of template RNA and numbers of PCR cycles. Based on these experiments, $5 \mu \mathrm{g}$ of total RNA from control tFKIF cells was used as a starting template for RT-PCR, and 25 cycles of PCR were performed. Data represent the mean of 3 independent experiments.

\section{TNF- $\alpha$ Treatment}

After 24-hour incubation in resting medium (DMEM supplemented with $0.5 \%$ FBS), $1 \times 10^{6}$ fibroblasts cultured in a $10-\mathrm{cm}$ culture dish were stimulated with $10 \mathrm{ng} / \mathrm{ml}$ recombinant human TNF- $\alpha$ (R\&D Systems, Minneapolis, Minn., USA) for $0,1,3,6$, and $24 \mathrm{~h}$. After the required period of stimulation, total RNA was extracted as described below.

\section{Contact Culture}

Prior to contact culture, PBMC were washed 3 times with PBS, and $1 \times 10^{7}$ cells were resuspended in $2 \mathrm{ml}$ of $10 \%$ FBS supplemented DMEM for $24 \mathrm{~h} .1 \times 10^{6}$ fibroblasts were cultured in a $10-\mathrm{cm}$ culture dish in resting medium for $24 \mathrm{~h}$. After centrifugation to separate the PBMC from the supernatant, $2 \mathrm{ml}$ of the supernatant was added to one fibroblast culture, and the pellet of PBMC resuspended in $2 \mathrm{ml}$ of the resting medium and overlaid on the other fibroblast culture. These contact culture experiments were performed for $0,1,3,6$, and $24 \mathrm{~h}$. Prior to extraction of total RNA from the fibroblasts, PBMC were removed by rinsing extensively with PBS until a few PBMC could be detected microscopically.

\section{Neutralizing Antibody Treatment}

After $24 \mathrm{~h}$ of resting, $1 \times 10^{6} \mathrm{tNKF}$ cells were pretreated with rabbit polyclonal anti-ICAM-1 or anti-VCAM-1 antibodies (5 and $50 \mu \mathrm{g} / \mathrm{ml}$; Chemicon International, Temecula, Calif., USA) for $30 \mathrm{~min}$. Then, the cells were washed with PBS, and the contact culture performed as described above.

\section{RNA Extraction and Semi-Quantitative RT-PCR}

Total cellular RNA was extracted with TRIZol (Gibco BRL) according to the manufacturer's instructions. In order to confirm that the RT-PCR products were within the linear amplification range, preliminary experiments were performed using various concentrations of template total RNA and numbers of PCR cycles (fig. 1). The RT-PCR parameters described below were selected because they gave a linear amplification range for the RT-PCR. In each experiment, $5 \mu \mathrm{g}$ of total RNA was reverse-transcribed to cDNA using Superscript II RT (Gibco BRL) in a $20-\mu \mathrm{l}$ RT reaction mixture. 
Fig. 2. MCP-1 gene expression in renal fibroblasts in response to TNF- $\alpha$ treatment. a tNKF cells. Sub-confluent tNKF cells were treated with $10 \mathrm{ng} / \mathrm{ml} \mathrm{TNF-} \alpha$ for $1,3,6$, and $24 \mathrm{~h}$. Expression of MCP-1 mRNA was increased significantly at $1,3,6$, and 24 h by TNF- $\alpha$. b tFKIF cells. TNF- $\alpha$ did not stimulate significantly sub-confluent tFKIF cells to express MCP-1 mRNA. Data represent the mean of 5 independent experiments.
Fig. 3. $\mathrm{MCP}-1$ gene expression in renal fibroblasts in response to the culture supernatant from PBMC. a tNKF cells. Sub-confluent tNKF cells were incubated with the supernatant for $1,3,6$, and $24 \mathrm{~h}$, and at $6 \mathrm{~h}$ tNKF cells expressed MCP-1 mRNA significantly. b tFKIF cells. The PBMC supernatant did not stimulate significantly sub-confluent tFKIF cells to express MCP-1 mRNA. Data represent the mean of 5 independent experiments.
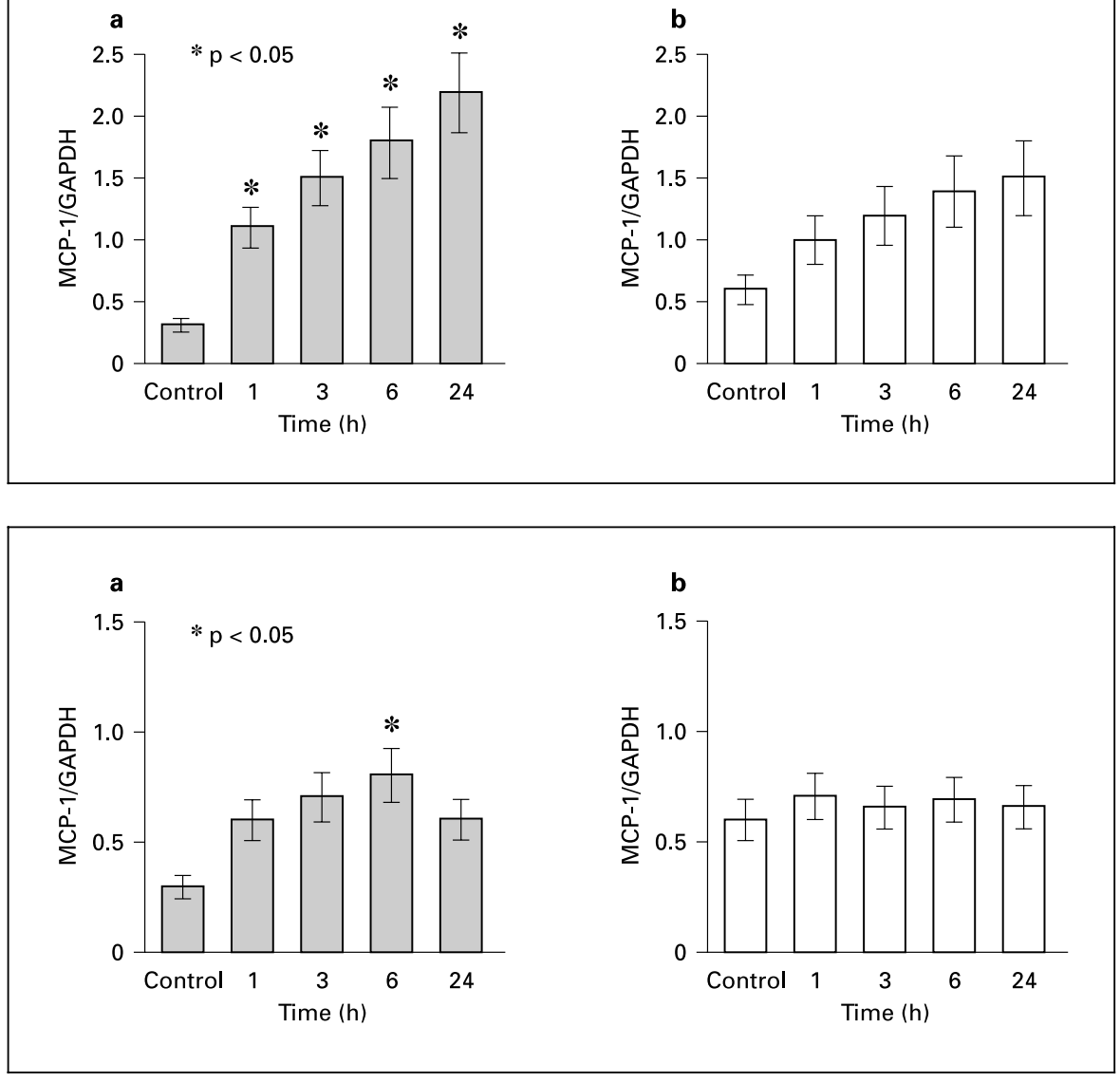

Then, $1 \mu 1$ of cDNA in the RT reaction mixture was used for the subsequent PCR. The PCR mixture consisted of $1.5 \mathrm{mM} \mathrm{MgCl}$, $50 \mathrm{~m} M \mathrm{KCl}, 10 \mathrm{~m} M$ Tris-HCl, pH 8.3, $0.25 \mathrm{~m} M$ each of dNTPs, $1 \mathrm{U}$ of Ampli Taq DNA polymerase (Takara, Tokyo, Japan), and forward and reverse primers (20 pmol each). The sequences of the primers were as follows: MCP-1, forward 5'-TTGTGTGCCTGCTGCTCATA-3' and reverse 5'-GGTTTGCTTGTCCAGGTGGT-3' (product size $263 \mathrm{bp}$ ), and glyceraldehyde-3-phosphate-dehydrogenase (GAPDH), forward 5'-ACCACAGTCCATGCCATCAC- ${ }^{\prime}$ and reverse 5'-TCCACCACCCTGTTGCTGTA-3' (product size $515 \mathrm{bp}$ ). The thermocycling conditions were 25 cycles of $95^{\circ} \mathrm{C}$ for $1 \mathrm{~min}$, $60^{\circ} \mathrm{C}$ for $30 \mathrm{~s}$, and $72^{\circ} \mathrm{C}$ for $1 \mathrm{~min}$. The PCR products were electrophoresed in a $2 \%$ agarose gel and visualized with ethidium bromide. Each band was scanned and analyzed by computer software, NIH image 1.6. Signal densities of MCP-1 mRNA were adjusted by GAPDH mRNA expression.

\section{Statistical Analysis}

All experiments were repeated 5 times. Data are presented as the mean \pm SE. Statistical significance was analyzed using Bonferroni/ Dunnett's test (ANOVA). $p$ values of $<0.05$ were considered statistically significant.

\section{Results}

\section{Treatment with TNF- $\alpha$}

In response to TNF- $\alpha$ stimulation, tNKF cells expressed MCP-1 mRNA at significant levels, reaching a peak at $24 \mathrm{~h}$ (fig. 2a). In tFKIF cells, the basal MCP-1 mRNA level was higher, but MCP-1 mRNA expression did not change significantly following TNF- $\alpha$ stimulation (fig. 2b).

\section{Treatment with Culture Supernatant}

The culture supernatant from PBMC significantly increased MCP-1 mRNA expression in tNKF cells, and the response reached a peak at $6 \mathrm{~h}$ (fig. 3a). On the other hand, tFKIF cells did not respond to the supernatant (fig. 3b).

\section{Contact Culture}

Direct contact with PBMC significantly increased MCP-1 mRNA expression in tNKF cells at 1, 3, and $6 \mathrm{~h}$, 
Fig. 4. MCP-1 gene expression in renal fibroblasts in response to direct contact with PBMC in contact-culture. a tNKF cells. Subconfluent tNKF cells were cultured in contact with PBMC for 1, 3, 6, and $24 \mathrm{~h}$. Expression of MCP-1 mRNA increased significantly in response to a direct contact with PBMC at 1,3 , and $6 \mathrm{~h}$. b tFKIF cells. The direct contact with PBMC could not significantly stimulate sub-confluent tFKIF cells to express MCP-1 mRNA. Data represent the mean of 5 independent experiments.

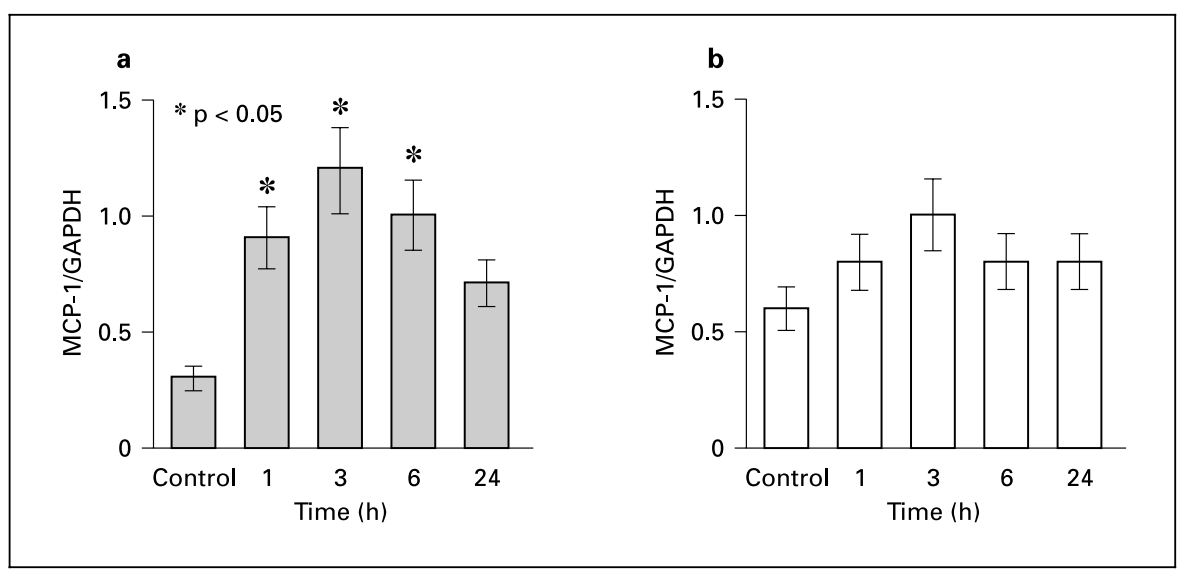

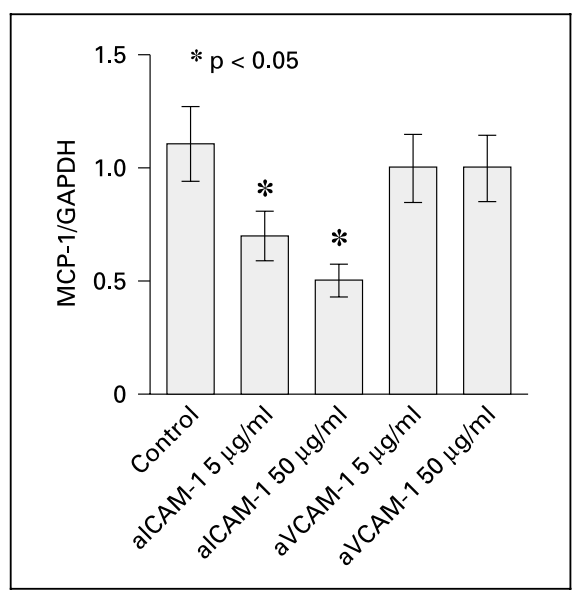

Fig. 5. Effects of neutralizing antibodies to ICAM- 1 and VCAM- 1 on MCP-1 mRNA expression in tNKF cells. After 30-min pretreatment with antibodies against ICAM-1 or VCAM-1, sub-confluent tNKF cells were cultured in contact with PBMC for $3 \mathrm{~h}$. AntiICAM-1 antibody, but not anti-VCAM-1 antibody, decreased significantly MCP-1 mRNA expression in tNKF cells cultured in contact with PBMC. Data represent the mean of 5 independent experiments.

and the peak level of the expression was at $3 \mathrm{~h}$ (fig. 4a). However, the expression of the MCP-1 gene in tFKIF cells was not stimulated during contact culture with PBMC (fig. 4b).

\section{Treatment with Neutralizing Antibody}

Pretreatment with anti-ICAM-1 antibody suppressed the increases in the expression of the MCP-1 gene in
tNKF cells stimulated by contact culture with PBMC for $3 \mathrm{~h}$ (fig. 5). In contrast, treatment with anti-VCAM-1 antibody had no effect (fig. 5).

\section{Discussion}

In this study, we demonstrated that the cell-to-cell interaction with PBMC could significantly increase MCP-1 gene expression in renal fibroblasts, suggesting that such an interaction is likely to be involved in the acceleration of immune cell infiltration into the renal interstitium. At the early stage of a given disease, after migration through the endothelium, the principle interaction of leukocytes is with normal fibroblasts in the kidney $[1,2,4,5]$. Activated PBMC produce a variety of humoral factors, including TNF- $\alpha$ [2], which could increase MCP-1 gene expression in renal fibroblasts without direct contact between the cells. Direct contact with PBMC, however, could significantly increase MCP-1 gene expression in renal fibroblasts, possibly through ICAM-1 activation via ligand-receptor binding and the unique signaling pathways within renal fibroblasts. ICAM- 1 is a member of the immunoglobulin superfamily, and forms the counter receptor for the leukocyte $\beta_{2}$-integrins $[5,6,13]$. The ligation of ICAM- 1 on endothelial cells by $\beta_{2}$-integrins mediates the firm adhesion of leukocytes to the endothelial lining of the vasculature at inflammatory sites. In addition to leukocyte adhesion and subsequent extravasation, Blaber et al. [5], Clayton et al. [6] and Clayton and Steadman [13] revealed that the ligation of ICAM-1 on renal fibroblasts activates AP- 1 and NF- $\mathrm{\kappa B}$ and positively regulates RANTES and ICAM-1 gene expression [5, 6, 13], amplifying inflammation. It is well known that the $5^{\prime}$ 
flanking region of the human $\mathrm{MCP}-1$ gene has an $\mathrm{NF}-\kappa \mathrm{B}$ element [14], which is required for transcription of the gene [15]. In addition, the involvement of adhesion molecules in the activation of NF- $\mathrm{KB}[16]$ and in the expression of the MCP-1 gene has been suggested $[17,18]$. Thus, in this study, ICAM-1 likely mediates MCP-1 gene expression in tNKF cells in the same manner.

It has been shown that human renal fibroblasts derived from a kidney with fibrosis (FKIF) exhibit an increased rate of spontaneous proliferation in vitro compared to fibroblasts from a normal kidney (NKF) [19]. Rodemann and Müller [20] and Lonnemann et al. [21] also reported that these fibroblasts had different cytokine response profiles: IL-1 $\beta$ stimulates the growth and collagen production of FKIF, but inhibits proliferation of NKF. In this study the constitutive level of MCP-1 mRNA in tFKIF cells was consistently higher than in tNKF cells, and the MCP-1 gene expression in tNKF cells, but not in tFKIF cells, increased significantly in response to humoral factors and a direct contact with PBMC. These differences in fibroblast phenotype can be carried over in an in vitro situation, possibly because a given, stable phenotype has been selected by the inflammatory/profibrotic environment in vivo [12, 22]. It seems reasonable to assume that NKF produce MCP-1 in response to direct contact with invading leukocytes and facilitate cellular infiltration, promoting interstitial fibrogenesis and developing to an FKIF phenotype, producing MCP-1 constitutively.
Experiments with neutralizing antibodies, antisense DNA oligonucleotides, and gene knock-out technology revealed that MCP-1 is involved in interstitial alterations in the nephritic kidney [8-10]. Recently, Wada et al. [7] found that MCP-1 likely plays a role in interstitial fibrosis in human diabetic nephropathy. Before drawing conclusions, we must draw attention to a limitation of our in vitro results stemming from stimulation within $24 \mathrm{~h}$, which might not directly be associated with the progression of renal fibrosis. We believe that infiltrating monocytes would make contact with and stimulate the renal fibroblasts to produce $\mathrm{MCP}-1$, which further recruit monocytes into the interstitium to communicate with other fibroblasts, thereby generating a vicious cycle. Those accumulated, activated fibroblasts and monocytes secret profibrotic molecules and facilitate renal fibrosis.

Taken together with the present findings, MCP-1 is a key player in the interstitial alterations in the kidney, and MCP-1 blockades, e.g., a p38 MAPK inhibitor [23], seem to be promising therapy to prevent the progression of a variety of kidney diseases.

\section{Acknowledgements}

We are grateful to J. Takahashi, S. Yamada, and M. Otobe for their technical assistance. L.H. was the recipient of a scholarship from the Sasagawa Memorial Foundation. A portion of this work was presented at the 45th Annual Meeting of the Japanese Society of Nephrology (2002, Osaka, Japan).

\section{References}

1 Okada H, Strutz F, Danoff TM, Kalluri R, Neilson EG: Possible mechanisms of renal fibrosis; in Koide H (ed): Progression of Chronic Renal Diseases. Contrib Nephrol. Basel, Karger, 1996, vol 118, pp 147-154.

2 Eddy A: Molecular basis of renal fibrosis. Pediatr Nephrol 2000;15:290-301.

3 Risdon R, Sloper J, De Vardener H: Relationship between renal function and histological changes found in renal-biopsy specimens from patients with persistent glomerulonephritis. Lancet 1968;ii:363-366.

4 Norman JT, Fine LG: Progressive renal disease: Fibroblasts, extracellular matrix, and integrins. Exp Nephrol 1999;7:167-177.

5 Blaber R, Stylianou E, Clayton A, Steadman R Selective regulation of ICAM-1 and RANTES gene expression after ICAM-1 ligation on human renal fibroblasts. J Am Soc Nephrol 2003; 14:116-127.

6 Clayton A, Evans RA, Pettit E, Hallett M, Williams JD, Steadman R: Cellular activation through the ligation of intercellular adhesion molecule-1. J Cell Sci 1998;111:443-453.
7 Wada T, Furuichi K, Sakai N, Iwata Y, Yoshimoto K, Shimizu M, Takeda S, Takasawa K, Yoshimura M, Kida H, Kobayashi K, Mukaida N, Naito T, Matsushima K, Yokoyama H: Upregulation of monocyte chemoattractant protein-1 in tubulointerstitial lesions of human diabetic nephropathy. Kidney Int 2000;58: 1492-1499.

8 Tesch GH, Schwarting A, Kinoshita K, Lan HY, Rollins BJ, Kelly VR: Monocyte chemoattractant protein-1 promotes macrophage-mediated tubular injury, but not glomerular injury, in nephrotoxic serum nephritis. J Clin Invest 1999;103:73-80

9 Okada H, Moriwaki K, Kalluri R, Imai H, Ban S, Takahama M, Suzuki H: Inhibition of monocyte chemoattractant protein-1 expression in tubular epithelium attenuates tubulointerstitial alteration in rat Goodpasture syndrome. Kidney Int 2000;57:927-936.
10 Lloyd CM, Minto AW, Dorf ME, Proudfoot A, Wells TNC, Salant DJ, Gutierrez-Ramos JC: RANTES and monocyte chemoattractant protein-1 (MCP-1) play an important role in the inflammatory phase of crescentic nephritis, but only MCP-1 is involved in crescent formation and interstitial fibrosis. J Exp Med 1997;185: 1371-1380.

11 Hisada Y, Sakurai H, Sugaya T: Cell to cell interaction between mesangial cells and macrophages induces the expression of monocyte chemoattractant protein-1 through nuclear factor- $\mathrm{\kappa B}$ activation. Biochem Biophys Res Commun 2000;269:309-316.

12 Muller GA, Frank J, Rodemann HP, EnglerBlum G: Human renal fibroblast cell lines (tFKIF and $\mathrm{tNKF}$ ) are new tools to investigate pathophysiologic mechanisms of renal interstitial fibrosis. Exp Nephrol 1995;3:127-133.

13 Clayton A, Steadman R: ICAM-1 interactions in the renal interstitium: A novel activator of fibroblasts during nephritis. Histol Histopathol 1999;14:861-870. 
14 Shyy YJ, Li YS, Kolattukudy PE: Structure of human monocyte chemotactic protein gene and its regulation by TPA. Biochem Biophys Res Commun 1990;169:346-351.

15 Ueda A, Okuda K, Ohno S, Shirai A, Igarashi T, Matsunaga K, Fukushima J, Kawamoto S, Ishgatsubo Y, Okubo T: NF-кB and Spl regulate transcription of the human monocyte chemoattractant protein-1 gene. J Immunol 1994; 153:2052-2063.

16 Marra F, Pastacaldi S, Romanelli M, Pinzani M, Ticali P, Carloni V, Laffi G, Gentilini P: Integrin-mediated stimulation of monocyte chemotactic protein-1 expression. FEBS Lett 1997;414:221-225.
17 Krushel LA, Chuuningham BA, Edelman GM, Crossin KL: NF- $\kappa B$ activity is induced by neural cell adhesion molecule binding to neurons and astrocytes. J Biol Chem 1999;274:24322439.

18 Scatena M, Almeida M, Chaisson ML, Fausto $\mathrm{N}$, Nicosia RF, GIachelli CM: NF- $\kappa \mathrm{B}$ mediates avb3 integrin-induced endothelial cell survival. J Cell Biol 1998;141:1083-1093.

19 Rodemann HP, Müller GA: Abnormal growth and clonal proliferation of fibroblasts derived from kidneys with interstitial fibrosis. Proc Soc Exp Biol Med 1990;195:57-63.

20 Rodemann HP, Müller GA: Characterization of human renal fibroblasts in health and disease: II. In vitro growth, differentiation, and collagen synthesis of fibroblasts from kidneys with interstitial fibrosis. Am J Kidney Dis 1991;17:684-686.
21 Lonnemann G, Shapiro L, Engler-Blum G, Müller GA, Koch KM, Dinarello CA: Cytokines in human renal interstitial fibrosis. I. Interleukin-1 is a paracrine growth factor for cultured fibrosis-derived kidney fibroblasts. Kidney Int 1995;47:837-844.

22 Grupp C, Müller G: Renal fibroblast culture. Exp Nephrol 1999;7:377-385.

23 Wada T, Furuichi K, Sakai N, Iwata Y, Yoshimoto K, Shimizu M, Kobayashi K, Mukaida N, Matsushima K, Yokoyama H: A new antiinflammatory compound, FR167653, ameliorates crescentic glomerulonephritis in WisterKyoto rats. J Am Soc Nephrol 2000;11:15341541 